\title{
Contactless Characterization of Metals Thermal Expansion Coefficient by a Free-Space RF Measurement
}

\author{
Florian Requena, Student Member, IEEE, Nicolas Barbot, Member, IEEE, \\ Darine Kaddour, Member, IEEE, and Etienne Perret, Senior Member, IEEE
}

\begin{abstract}
In this paper, a novel method to characterize the thermal dilatation of metals using a contactless measurement of the frequencydependant reflection coefficient of a resonator is introduced. This wireless technique allows extracting the thermal expansion coefficient of the material without the need of any calibration. Two antennas, a VNA and a scatterer fabricated with the under-test material are required. All the measurements have been achieved using a climatic chamber with absorbers to control the scatterer's temperature. Measured values for thermal expansion coefficients are in good agreement with the values provided by the manufacturer which is confirmed by a sensibility study.
\end{abstract}

Index Terms-Contactless measurement, RF characterization, Scatterer, Thermal expansion

\section{INTRODUCTION}

$\mathbf{T}$ HERMAL expansion coefficient is a crucial parameter for applications in the field of civil engineering or semiconductor industries. We can see in the literature that many different experimental approaches have been used. This significant diversity comes from the great variety of techniques that exist to measure displacement. We can cite purely mechanical approaches, or the very wide variety of optical approaches (interferometry, optical imaging, $\mathrm{x}$-rays diffraction...) [1]. Thus, to extract the thermal expansion of a material, examination of the literature reveals for example mechanical dilatometers such as capacitance dilatometers [2] which consist of two parallel plates with one stationary and one that can move. The thermal expansion moves the free plate and so, shifts the gap between the plates. By measuring the capacitance, the thermal expansion coefficient can be extracted. Push-rod dilatometer [3] can also be found, where a rod of the material under-test expands and transfers its expansion to a pressure gage measuring the displacement. This method is widely used for many reasons. For example, it is compatible with most materials. However, one of the main drawbacks of this test bench comes from the fact that the material under test is in contact with the bench, so particular attention must be paid to thermally isolate the bench from the heated sample. Specific materials are needed to fabricate the test bench, and reference samples can be used to reduce the uncertainty. For this reason, contactless methods have been widely studied. To develop more precise test bench, several optical approaches have been used to measure CTE [4]. Compared to dilatometry, significantly greater accuracy can be obtained. For example, optical dilatometers using an interferometer can have resolution of $0.1 \%$ [5], [6]. However, the main limitation of these techniques, such as interferometry, is a maximum temperature of measurement above $700^{\circ} \mathrm{C}$. Other drawbacks of these methods are the cost of optical setup, the need of alignment accuracy as well as a strict control of the environment (the use of a vacuum chamber for the effect of the refractive index of the atmosphere on the wavelength of light, strict control of the wavelength of the laser...). Another optical method consists in a digital microscope to picture the expansion as a function of temperature and an optical mire to

The authors are with Univ. Grenoble Alpes, Grenoble INP, LCIS, F-26000 Valence, France.

E. Perret is also with Institut Universitaire de France, Paris, France. convert pixel to length [7]. For some materials such as crystals, Xray diffraction can be used to measure the crystal lattice parameter as a function of the temperature in order to extract its thermal expansion [8]. From the state of the art, each experimental approach has advantages and disadvantages depending on a lot of factors such as the uncertainty, the sample size or shape, the sample preparation time, or the temperature range required. Note also that most of these test methods have been standardized for general use [9], [10], [11]. A brief summary of the general advantages and disadvantages of some approaches is given in Table I. Multiple other CTE measurement benches exist and have been listed in [1].

In the first raw of Table I, the uncertainty of the different methods is compared based on some references in order to give examples of the error that can be reached. However, even if the CTE of various materials are listed in a large number of reference books, the uncertainty is seldom reported. Due to the fact that these values are averaged over large ranges of temperature in most sources, the uncertainty at ambient temperature can be increased. Another reason why reference books could not be accurate enough is relating to the fact that metals are rarely pure, so CTE can vary from manufacturer to manufacturer. Note also that all these different experimental approaches require dedicated and optimised test benches that are generally not commercially available or not easy to access to non-expert in the field of materials engineering. For all these reasons, an easy approach to implement CTE measurement technique is required. Contrary to most existing techniques, the one presented here requires no calibration or sample of reference. It is based on a very simple analytical model that everyone can implement. Moreover, the bench is composed of commercially available off-the-shelf RF equipment. Last but not least, this novel test bench can be easily replicated by any expert in the field of RF in order to measure the CTE of his own material.

Despite the very large number of test benches developed for the measurement of CTE, to our knowledge, none is based on an RF principle. However, many years ago it has been shown that by sending an electromagnetic wave towards a specific target, and by receiving the backscattered field, many physical quantities can be sensed wirelessly. A striking example of such back-scattering application can be traced back to the early 1900's with Leon Theremin's work [12]. This work includes the contactless musical instrument called 'Thereminvox' which uses the position of the hand to produce a sound, or the passive listening device called 'The Great Seal bug' [12]. Since then, numerous works have been done to wirelessly sense physical quantities using this back-scattering principle such as mechanical displacement of the order of $100 \mu \mathrm{m}$ [13], strain [14], small variations due to the fabrication randomness of the tag for authentication [15], or even permittivity [16]. The closeness between $\mathrm{RF}$ and thermal domain has been also used to develop RF temperature sensors by using, for example, temperature dependence of permittivity [17] or dilatation of a 3D structure [18]. Here, a free-space measurement of the resonance frequency of a resonator is used to characterize the thermal coefficient of metals. Specific planar resonant 
TABLE I

AdVANTAGES AND DISADVANTAGES OF EXISTING METHODS

\begin{tabular}{|c|ccccc|}
\hline & $\begin{array}{c}\text { Mecanical } \\
\text { (push-rod) }\end{array}$ & $\begin{array}{c}\text { Optical } \\
\text { (microscope) }\end{array}$ & $\begin{array}{c}\text { Optical } \\
\text { (interference) }\end{array}$ & X-ray & This paper \\
\hline Error on CTE & $4 \%[19]$ & $6 \%[7]$ & $<1 \%[20]$ & $6 \%[21]$ & $6 \% *$ \\
Materials adaptability & Very good & Good & Good & Only crystals & Only metals \\
Contactless & No & Yes & Depend & Yes & Yes \\
Alignment accuracy & Not needed & Needed & Needed & Needed & Not needed \\
Sample preparation & Minimal & - & Depend & Extensive & Medium \\
Equipment cost & Low & Medium & High & High & Medium \\
Standardized & {$[9]$} & No & {$[10]$} & No & No \\
\hline
\end{tabular}

* An error lower than $1 \%$ has been obtained with Zinc and Copper.

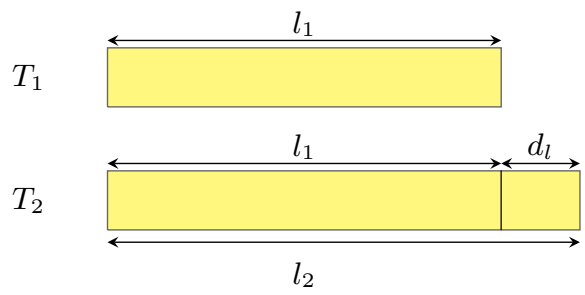

Fig. 1. Expansion of a 1D object due to temperature variation.

scatterers fabricated with several metals under characterization have been introduced for this purpose. The scatterer's shape is simple enough to be modeled with an analytical expression and also to fabricate. With this model, the derivation of the CTE does not require any advanced computation, any RF solver or even a lookup table as it is generally the case when one seeks to capture a physical quantity by using the principle of back-scattering because of the complexity of the problem. The measurement technique is essentially based on the resonator which has been designed to meet precise specifications: the resonator is made entirely of metal, in one layer and has a high resonating behavior. As described below, these two characteristics are essential for the measurement of CTE using RF waves.

\section{THERMAL DILATATION}

The thermal expansion of a material is defined as the modification of its volume with a temperature variation at a constant pressure. If we consider a 1-dimension object of length $l_{1}$ at temperature $T_{1}$ (Fig. 1), this object will expand with an increase of temperature, and the final length at a temperature $T_{2}>T_{1}$ is :

$$
l_{2}=l_{1}+d_{l}=l_{1}\left(1+\alpha\left(T_{2}-T_{1}\right)\right)
$$

where $d_{l}$ is the expansion in length due to the increase in temperature [1] and $\alpha$ the coefficient of thermal expansion (CTE) of the material. $T_{2}-T_{1}$ will be written $\Delta T$ in the rest of the paper.

In this work, the thermal expansion coefficient $\alpha$ is supposed to be temperature-independent in the considered temperature range. Note that this coefficient is positive for almost all materials. In addition, this thermal effect affects a real 3D object in all directions which means that if the material is not isotropic for this phenomenon, the CTE can be different in the different directions $(x, y$, and $z)$.

In this paper, a loop resonator is considered as a scattering device and its thermal expansion is studied (Fig. 2). The resonator is only composed of one layer of metal. Due to the absence to any ground plane, the scatterer can simply be placed on top of any substrate. In such a case, the introduced model can be used as long as the substrate permittivity can be considered as temperature independent in the considered range. With this loop shape, the resonance frequency can be considered to be related only with its larger dimensions. So the extracted CTE is in the direction of the notch characterized by its

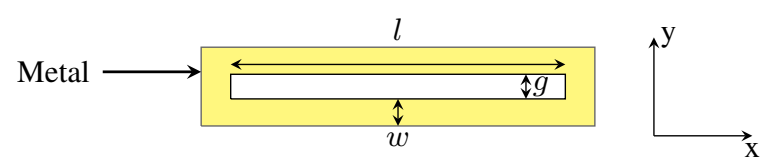

Fig. 2. Nominal dimensions of the fabricated loop (i.e. values used for the fabrication): $l=50.83 \mathrm{~mm}, g=2.07 \mathrm{~mm}$ and $w=1.43 \mathrm{~mm}$.

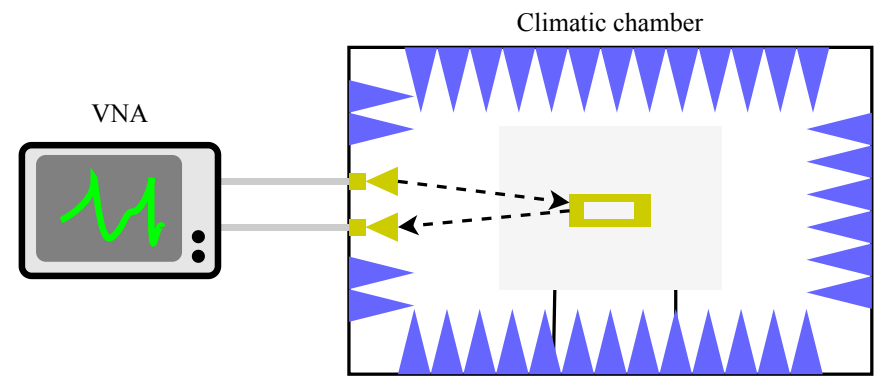

Fig. 3. Principle of the measurement of the resonance frequency used to extract the CTE.

length $l$ (along $\mathrm{x}$ axis) as illustrated in Fig. 2. In the rest of the paper, a physical quantity with subscript 1 (respectively 2 ) is considered at temperature $T_{1}$ (respectively $T_{2}$ ).

The measurement principle based on free-space measurement is illustrated in Fig. 3. When a loop is illuminated by an incoming electromagnetic-field, the maximum energy back-scattered at a temperature $T_{1}$ occurs at its resonant frequency $f_{1}$ defined by [22]:

$$
f_{1}=\frac{c}{2 \sqrt{\varepsilon_{e f f}}\left(l_{1}+2 \Delta l_{1}\right)}=\frac{c}{2 \sqrt{\varepsilon_{e f f}} L_{1}}
$$

where $c$ is the speed of light in vacuum, $\varepsilon_{e f f}$ is the effective permittivity seen by the loop, $l_{1}$ is the length of the notch and $\Delta l_{1}$ is the additional length taking into account the presence of short circuit (SC) discontinuities. Indeed the loop resonator can be considered as a transmission line section terminated at both ends by a SC [22]. Note that the effective length $L_{1}=l_{1}+2 \Delta l_{1}$ will be used for the rest of the paper. Note also that if the loop is used without any substrate, $\varepsilon_{\text {eff }}$ is equal to one.

As mentioned, the physical length of the resonator $l_{1}$ follows (1) but $2 \Delta l_{1}$ which physically corresponds to an interconnection realized by a conductor does not necessarily follow the same dependence with temperature. Indeed, the shape of this conductor is not only along the $x$ axis but also the $y$ axis. At a temperature $T_{2}>T_{1}$, the effective length $L_{1}$ will increase and its expression $L_{2}$ can be written as :

$$
L_{2}=l_{1}\left(1+\alpha_{c} \Delta T\right)+2 \Delta l_{1}\left(1+K \alpha_{c} \Delta T\right)
$$

with a constant coefficient $K$, where in the proposed model, describes the specific dilatation of the SC discontinuities. Therefore, the resonant frequency $f_{2}$ for a temperature $T_{2}>T_{1}$ will be shifted to lower frequencies and can be written as :

$$
f_{2}=\frac{c}{2 \sqrt{\varepsilon_{e f f}} L_{2}}=\frac{c}{2 \sqrt{\varepsilon_{e f f}} L_{1}(1+\beta \Delta T)}
$$

where $\beta$ is introduced as the equivalent expansion coefficient for the effective length $L_{1}$. The expression of $\beta$ is deduced from (3) and (4) and is :

$$
\beta=\alpha \frac{l_{1}+2 K \Delta l_{1}}{l_{1}+2 \Delta l_{1}}
$$

In order to study the importance of $K$, electromagnetic simulations of a loop resonator in vacuum with dimensions given in Fig. 2 have been carried out using the software CST MW. The quantity $2 \Delta l_{1}$ 


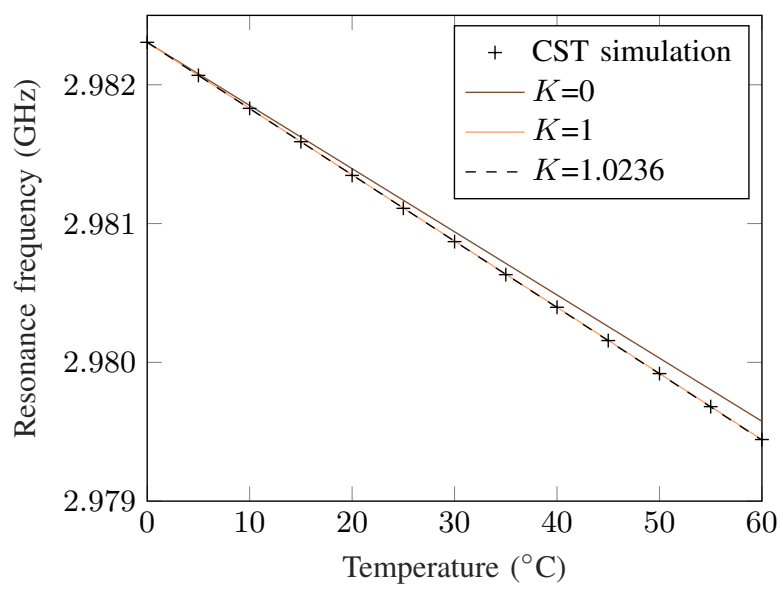

Fig. 4. Resonance frequencies of a loop versus the temperature. Comparison between simulations results and the introduced model (5) for different values of the coefficient $K$.

has been determined so that the effective length in (4) matches the simulated resonance frequency for $T=0^{\circ} \mathrm{C}$. The frequency response of the resonator has been extracted for different temperatures in the range $0-60^{\circ} \mathrm{C}$ based on (1). Fig. 4 presents the variation of the simulated resonant frequency versus temperature.

To illustrate the effect of $K$, with the use of (5), two cases were plotted: $K=0$ ( $\Delta l_{1}$ doesn't expand with temperature) and $K=1$ (both lengths are affected in the same way).

We can see that the case $(K=0)$ leads to the maximum error with the simulations while the case $(K=1)$ gives better results. However, the optimal result is obtained when $K=1.02$ leading to an equivalent expansion coefficient :

$$
\beta=1.01 \alpha
$$

The quantity $K$ is a geometry dependant parameter of the scatterer and $\Delta l_{1}$ is a function of the effective permittivity as expressed in [22]. Simulations with different loop dimensions $l$ show that $K$ can have different values (for example, $K=1.60$ for $l=39 \mathrm{~mm}$ ) which makes (6) necessary to obtain a good accuracy on the extracted CTE. A study of the influence of $K$ on the accuracy of the measurement is done in section IV.

When temperature increases, the effective length $L_{1}(1+\beta \Delta T)$ increases leading to a lower value of the resonant frequency as described by (4). If the substrate permittivity is assumed temperatureindependent, the value of $\beta$ can be extracted with the measurement of two resonant frequencies $\left(f_{1}, f_{2}\right)$ at different temperatures $\left(T_{1}\right.$, $T_{2}$ respectively) and then the value of $\alpha$ can be extracted using (6). Indeed, based on (4) we can write for two temperatures $T_{1}, T_{2}$ with a reference length $L_{0}$ at temperature $T_{0}$ :

$$
\begin{aligned}
& L_{1}=L_{0}\left(1+\beta\left(T_{1}-T_{0}\right)\right)=\frac{c}{2 \sqrt{\varepsilon_{e f f}} f_{1}} \\
& L_{2}=L_{0}\left(1+\beta\left(T_{2}-T_{0}\right)\right)=\frac{c}{2 \sqrt{\varepsilon_{e f f}} f_{2}}
\end{aligned}
$$

Considering (7) and (8), the CTE can be extracted using the following formula:

$$
\beta=\frac{f_{1}-f_{2}}{f_{2} T_{2}-f_{1} T_{1}-\left(f_{2}-f_{1}\right) T_{0}}
$$

Note that (9) is independent of $L_{0}$ and also of the effective permittivity $\varepsilon_{\text {eff }}$. With this method, the CTE can be extracted with a good accuracy without a prior knowledge on the length $l_{0}$ and effective permittivity of the scatterer. For the rest of the paper, the reference temperature $T_{0}$ is set to $0^{\circ} \mathrm{C}$ to be able to compare our

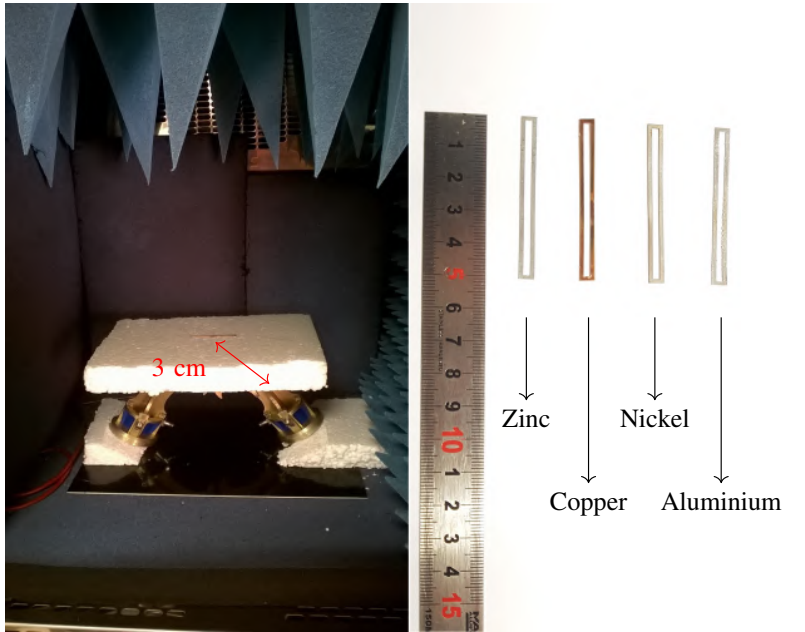

Fig. 5. Setup configuration. a) Setup inside the climatic chamber. b) The loop resonators made with different metals.

results with the data given by the metal's provider (Table II). In this case, (9) can be simplified:

$$
\beta=\frac{f_{1}-f_{2}}{f_{2} T_{2}-f_{1} T_{1}}
$$

Another solution to extract $\beta$ is to develop (7) and (8) using Taylor series of first order and to note that the frequency shift $\Delta f$ caused by a variation of the temperature is equal in first approximation to

$$
\Delta f \simeq f_{1} \beta\left(T_{2}-T_{1}\right)
$$

So if we have several measurements at different temperatures, this linear expression can be used to extract $\beta$.

\section{MeAsurements}

The measurement configuration setup and the fabricated tags used to determine the CTE are illustrated in Fig. 5. Tag information is given in Fig. 2. As shown in Fig. 3, tag support is polystyrene with an effective permittivity close to 1 . Based on results described in the previous section, a value of $K=1.02$ was considered to extract the CTE [see (6)].

A climatic chamber VC0018 by Votsch is used to control the temperature. Absorbers are placed inside the chamber to reduce the reflection level (see Fig. 3). A bi-static antenna configuration is used to increase the isolation. The protocol followed to do the measurements is the following: the temperature in the climatic chamber is first set. When the temperature is stabilized, the $S_{21}$ parameter is measured using a VNA. The exact measurement of the temperature of the loop is then determined using a thermal camera (Infrared Thermal Camera TC-1). We used this method to be sure of the extracted temperature because of the thermal inertia of the different materials inside the cavity. The measurements have been smoothed in order to remove any residual measurement noise that may impact the search for the maximum and thus induce an error on the frequency extraction. Raw and smoothed measurements are plotted in Fig. 7-b).

\section{RESULTS AND ANALYSIS}

The loop of the same geometrical dimensions presented in Fig. 2 has been realized with 4 different metals (Zinc, Nickel, Copper, and Aluminum). High purity metals have been chosen for the fabrication (more than 99\% purity). The CTE of these metals is given by the manufacturer following ASTM or DIN (international 


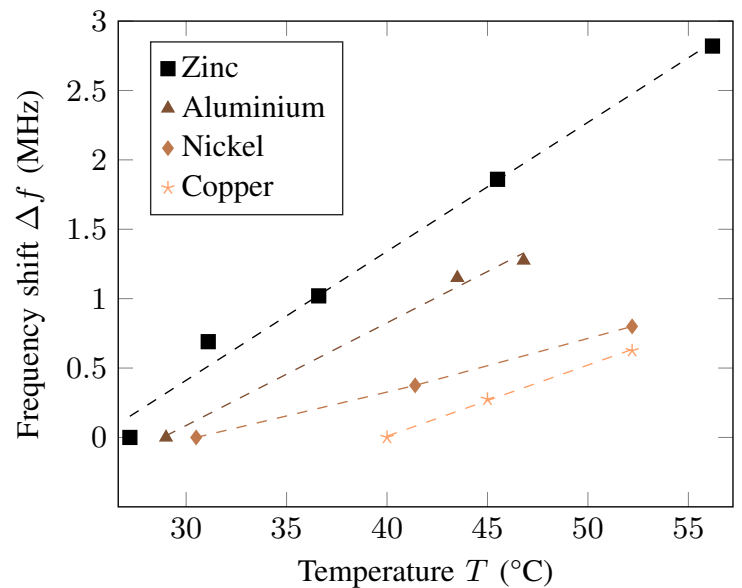

Fig. 6. Measured frequency shift versus temperature for the different metals.

TABLE II

Comparison of the CTE $(\alpha)$ Measured in RF AND Given By the PROVIDER

\begin{tabular}{|c|c|c|c|c|c|}
\hline & \multicolumn{3}{|c|}{$\begin{array}{c}\text { CTE } \\
\left(\times 10^{-6{ }^{\circ}} \mathrm{C}^{-1}\right)\end{array}$} & \multicolumn{2}{c|}{$\begin{array}{c}\text { Error in \% } \\
\text { with provider values }\end{array}$} \\
Metal & Provider & using (11) & using (10) & with (11) & with (10) \\
\hline Zinc & 31.0 & 31.03 & 31.08 & 0.09 & 0.25 \\
Copper & 17.0 & 16.96 & 17.13 & 0.2 & 0.76 \\
Nickel & 13.3 & 12.43 & 12.45 & 6 & 6 \\
Aluminium & $22-25$ & 22.1 & 22.07 & - & - \\
\hline
\end{tabular}

standards test-methods) [23] presented in Table II and compared to our measurements. Note that aluminium is coming from a different provider and its CTE is given with a precision of $\pm 5 \%$.

The zinc loop resonator has been measured for several temperatures from $27.2^{\circ} \mathrm{C}$ to $56.2^{\circ} \mathrm{C}$. The measured $S_{21}$ parameters are plotted in Fig. 7-a. Simulation results are also given for comparison. Note that a notch $l$ equal to $51.1 \mathrm{~mm}$ at $T=27.2^{\circ} \mathrm{C}$ has been considered in simulation to perfectly fit the data with the measurement results.

As shown in Fig. 7-a, simulations and measurements are in good agreement for the considered temperature range. A maximum error of $0.3 \mathrm{MHz}$ can be observed at $31.1^{\circ} \mathrm{C}$. With the exception of this temperature the measured resonance frequencies (Fig. 6) tend to a linear evolution as obtained in simulation and stated by (11).

By applying the protocol described in the previous section, the CTE of zinc can be extracted using (6). By considering only the minimum and maximum temperature values, a CTE of $31.08 \times 10^{-6}{ }^{\circ} \mathrm{C}^{-1}$ is computing using (10). A linear fitting based on the five different temperatures and by using using (11) leads to a value of $31.03 \times$ $10^{-6}{ }^{\circ} \mathrm{C}^{-1}$ presenting thus a relative error of $0.09 \%$ when compared to the value provided by the manufacturer.

Same measurement protocol has been applied for the three other metals (Copper, Nickel, Aluminum). The extracted CTE exhibits a good agreement with the value provided by the manufacturer as illustrated in Table II. For Copper and Nickel, both the fitting technique and the linear extraction give the same value for the CTE. The error compared to the provider value is higher for Nickel which presents the lowest CTE value. The lower is the CTE, the smaller is the expansion, and thus, accuracy is lower. For the lowest CTE (Nickel), an error of $6 \%$ is obtained while Zinc and Copper provide a better agreement with an error less than $1 \%$.

The relation between the measured expansion in length of the
TABLE III

UNCERTAINTIES

\begin{tabular}{|c|c|c|}
\hline Metal & $\begin{array}{c}\Delta \alpha \text { between measurement } \\
\text { and provider's value }\end{array}$ & $\Delta \alpha$ using (12) \\
\hline Zinc & $0.03 \times 10^{-6}$ & $1.9 \times 10^{-6}$ \\
Copper & $0.04 \times 10^{-6}$ & $3.35 \times 10^{-6}$ \\
Nickel & $0.87 \times 10^{-6}$ & $0.7 \times 10^{-6}$ \\
Aluminium & - & $3 \times 10^{-6}$ \\
\hline
\end{tabular}

loop $\Delta l$ due to an increase of temperature is plotted in Fig. 8. It is interesting to note that we can extract from this figure that the approach is accurate enough to measure dimension variation of $\Delta l=$ $3.6 \mu \mathrm{m}$. This is observable for aluminium between $43^{\circ} \mathrm{C}$ and $46^{\circ} \mathrm{C}$. This value can be compared with the $25 \mu \mathrm{m}$ of variation in length already detected based on a RF backscattering wave approach in [15]. Fig. 8 shows the high sensitivity to length variation of the proposed approach which is requested to extract with high accuracy the CTE of different metals.

In Fig. 6 the measured frequency of the different metals as a function of temperature is plotted. Note that, in such representation, the slope of the measured frequency shift is equal to $f_{0} \beta$ as stated by (11).

An analysis of the evaluation of $K$ on the extracted CTE can be done in order to verify the criticality of this parameter. We can compute the CTE $(\alpha)$ by using (5). By using this equation, we can vary the value of $K$ to evaluate its effect on the extraction of $\alpha$, for example if a constant value of $K$ is used for several loop dimensions.

If we vary the value of $K$ from 0.6 to 1.6 , we see that an error of $2 \%$ is obtained in the worst case if $K$ is equals to 1.6. This means that an error of $56 \%$ on $K$ only introduces an error of $2 \%$ on the measured CTE.

\section{UNCERTAINTY OF THE METHOD}

The theoretical uncertainty of the method can be estimated. From (6) and (10), the error on $\alpha$ can be written as

$$
\begin{aligned}
1.01 \Delta \alpha & =\frac{f_{2}\left(T_{2}-T_{1}\right)}{\left(f_{2} T_{2}-f_{1} T_{1}\right)^{2}} \Delta f_{1}+\frac{f_{1}\left(T_{1}-T_{2}\right)}{\left(f_{2} T_{2}-f_{1} T_{1}\right)^{2}} \Delta f_{2} \\
& +\frac{f_{1}\left(f_{1}-f_{2}\right)}{\left(f_{2} T_{2}-f_{1} T_{1}\right)^{2}} \Delta T_{1}+\frac{f_{2}\left(f_{2}-f_{1}\right)}{\left(f_{2} T_{2}-f_{1} T_{1}\right)^{2}} \Delta T_{2}
\end{aligned}
$$

By considering a resolution of $10 \mathrm{kHz}$ on the frequency measurement and an error of $1{ }^{\circ} \mathrm{C}$ on the measured temperature, sensitivities are computed and the results are given in Table III.

This sensibility study shows that in order to have a better precision for CTE extraction, a higher variation in temperature is required. The higher is the difference, the higher is the resolution on the extracted CTE. Also (12) can be applied to have an idea of the uncertainty on the extracted value. If a lower precision is required, the temperature variation range during the measurements can be increased.

\section{CONCLUSION}

In this paper, a novel method based on a contactless measurement approach to extract metals CTE is introduced. A method that permits to extract this coefficient with better precision than the one used to fabricate the planar resonator as discussed. This approach allows a wireless measurement with no calibration needed. Resonator loops on several metals like Zinc, Nickel, Copper and Aluminum have been realized and tested. Extracted values for thermal expansion coefficient are in good agreement with the values provided by the manufacturer, 

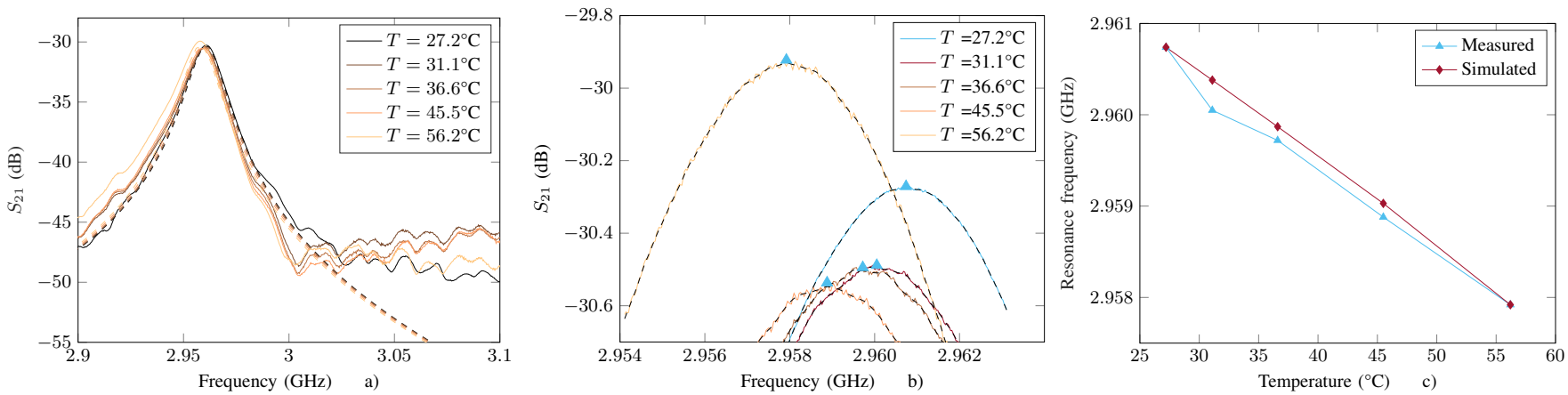

Fig. 7. a) Measured and simulated $S_{21}$ response for a zinc loop versus frequency for several values of temperatures - Measurement (continuous line) and simulation (dashed line). b) Measured and smoothed $S_{21}$ response for a zinc loop - Smoothed measurement (dashed line) and raw data (continuous line). c) Extraction of the resonance frequencies smoothed and simulated.

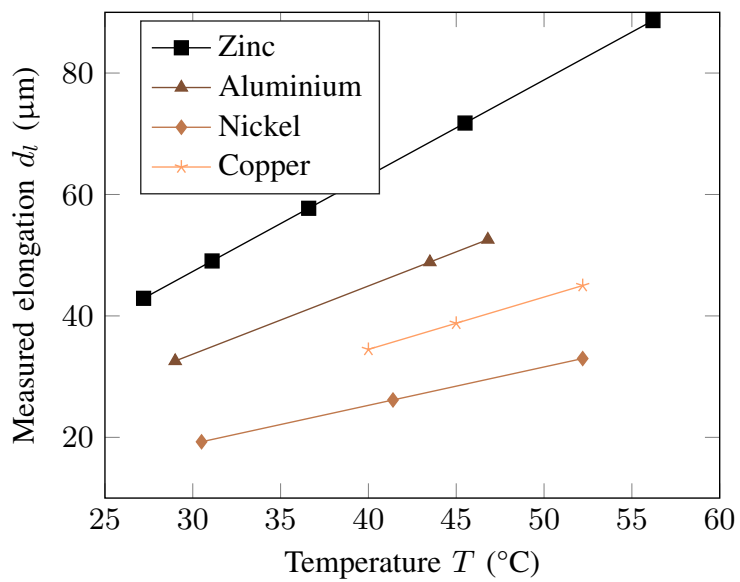

Fig. 8. Measured length elongation versus temperature for the different metals from the reference temperature $T_{1}=0^{\circ} \mathrm{C}$. The measured elongation is obtained using the fitted $\beta$ of Fig. 6 .

highlighting thus the potential of the free-space measurement approach for the thermal expansion coefficient characterization.

\section{ACKNOWLEDGMENT}

This project has received funding from the European Research Council (ERC) under the European Union's Horizon 2020 research and innovation program (grant agreement No 772539 ScattererID). This work is also supported by Univ. Grenoble Alpes.

\section{REFERENCES}

[1] J. James, J. Spittle, S. Brown, and R. Evans, "A review of measurement techniques for the thermal expansion coefficient of metals and alloys at elevated temperatures," Measurement science and technology, vol. 12, no. 3, p. R1, 2001.

[2] J. Neumeier, R. Bollinger, G. Timmins, C. Lane, R. Krogstad, and J. Macaluso, "Capacitive-based dilatometer cell constructed of fused quartz for measuring the thermal expansion of solids," Review of Scientific Instruments, vol. 79, no. 3, p. 033903, 2008.

[3] G. R. Hyde, L. P. Domingues, and L. R. Furlong, "Improved dilatometer," Review of Scientific Instruments, vol. 36, no. 2, pp. 204-205, 1965.

[4] E. G. Wolff, "Fundamentals of optical interferometry for thermal expansion measurements," in Proceedings of the 27th International Thermal Conductivity Conference and the 15th International Thermal Expansion Symposium, 2003, pp. 615-633.

[5] G. Bianchini, M. Barucci, T. Del Rosso, E. Pasca, and G. Ventura, "Interferometric dilatometer for thermal expansion coefficient determination in the 4-300K range," Measurement Science and Technology, vol. 17, no. 4 , p. $689,2006$.
[6] C. G. Tseng and Y. S. Jiang, "Optical interference dilatometer," Measurement Science and Technology, vol. 16, no. 10, p. 2114, 2005.

[7] R. Montanini and F. Freni, "Non-contact measurement of linear thermal expansion coefficients of solid materials by infrared image correlation," Measurement Science and Technology, vol. 25, no. 1, p. 015013, 2013.

[8] Y. Okada and Y. Tokumaru, "Precise determination of lattice parameter and thermal expansion coefficient of silicon between 300 and 1500K," Journal of applied physics, vol. 56, no. 2, pp. 314-320, 1984.

[9] "Standard test method for linear thermal expansion of solid materials with a vitreous silica dilatometer," E 228-95, annual book of astm standards, astm, 1995."

[10] "Standard test method for linear thermal expansion of rigid solids with interferometry," E 289-99, annual book of astm standards, astm, 1999."

[11] "Standard test method for linear thermal expansion of solid materials by thermomechanical analysis," E 831, annual book of astm standards, astm, 2000."

[12] P. Nikitin, "Leon Theremin (lev termen)," IEEE Antennas and Propagation Magazine, vol. 54, no. 5, pp. 252-257, 2012.

[13] E. Perret, "Displacement sensor based on radar cross-polarization measurements," IEEE Trans. Microw. Theory Techn., vol. 65, no. 3, pp 955-966, 2017.

[14] T. T. Thai, H. Aubert, P. Pons, G. DeJean, M. M. Tentzeris, and R. Plana, "Novel design of a highly sensitive RF strain transducer for passive and remote sensing in two dimensions," IEEE Transactions on Microwave Theory and Techniques, vol. 61, no. 3, pp. 1385-1396, 2013.

[15] Z. Ali, N. Barbot, R. Siragusa, E. Perret, D. Hely, and F. Bernier, Maxime Garet, "Detection of minimum geometrical variation by freespace-based chipless approach and its application to authentication," IEEE Microw. Wireless Compon. Lett., vol. 28, no. 4, pp. 323-325, 2018.

[16] F. Costa, A. Gentile, S. Genovesi, L. Buoncristiani, A. Lazaro, R. Villarino, and D. Girbau, "A depolarizing chipless RF label for dielectric permittivity sensing," IEEE Microw. Wireless Compon. Lett., vol. 28, no. 5, pp. 371-373, 2018.

[17] E. M. Amin and N. Karmakar, "Development of a chipless RFID temperature sensor using cascaded spiral resonators," in IEEE SENSORS 2011, 2011, pp. 554-557.

[18] T. T. Thai, F. Chebila, J. M. Mehdi, P. Pons, H. Aubert, G. R. DeJean, M. M. Tentzeris, and R. Plana, "Design and development of a millimetrewave novel passive ultrasensitive temperature transducer for remote sensing and identification," in the 40th European Microwave Conference, 2010, pp. $45-48$.

[19] J. Valentich, "A vitreous silica tube dilatometer for the measurement of thermal expansion of solids from- 195 to $1000^{\circ} \mathrm{C}$," Journal of Materials Science, vol. 14, no. 2, pp. 371-378, 1979.

[20] M. Okaji and H. Imai, "A practical measurement system for the accurate determination of linear thermal expansion coefficients," Journal of Physics E: Scientific Instruments, vol. 17, no. 8, p. 669, 1984.

[21] T. R. Touloukian YS, Kirby RK and D. PD, Thermophysical Properties of Matter, Volume 12-Thermal Expansion, Metallic Elements and Alloys. New York: IFI-Plenum, 1975.

[22] O. Rance, R. Siragusa, P. Lemaître-Auger, and E. Perret, "Contactless characterization of coplanar stripline discontinuities by RCS measurement," IEEE Trans. Antennas Propag., vol. 65, no. 1, pp. 251-257, 2016.

[23] Goodfellow.com. (2019). [accessed 28 oct. 2019]. [Online]. Available: http://www.goodfellow.com/PDF/TAB004F.pdf 